\title{
Inducing an Optical Feshbach Resonance via Stimulated Raman Coupling
}

\author{
Gregor Thalhammer, ${ }^{1}$ Matthias Theis, ${ }^{1}$ Klaus Winkler, ${ }^{1}$ Rudolf Grimm, ${ }^{1,2}$ and Johannes Hecker Denschlag ${ }^{1}$ \\ ${ }^{1}$ Institut für Experimentalphysik, Universität Innsbruck, Technikerstraße 25, 6020 Innsbruck, Austria \\ ${ }^{2}$ Institut für Quantenoptik und Quanteninformation, Österreichische Akademie der Wissenschaften, 6020 Innsbruck, Austria
}

(Dated: August 14, 2018)

\begin{abstract}
We demonstrate a novel method of inducing an optical Feshbach resonance based on a coherent free-bound stimulated Raman transition. In our experiment atoms in a ${ }^{87} \mathrm{Rb}$ Bose-Einstein condensate are exposed to two phase-locked Raman laser beams which couple pairs of colliding atoms to a molecular ground state. By controlling the power and relative detuning of the two laser beams, we can change the atomic scattering length considerably. The dependence of scattering length on these parameters is studied experimentally and modelled theoretically.
\end{abstract}

PACS numbers: 34.50Rk, 32.80.Pj, 03.75.Nt, 34.20.Cf

\section{INTRODUCTION}

Feshbach resonances have become a central tool in the physics of ultra-cold quantum gases during the last years because they allow for a tuning of the interactions between atoms. Controlling interparticle interactions is a central key in many fields of modern physics and is especially relevant for future applications in quantum computation and exploring novel many-particle quantum effects. Beautiful experiments using magnetically tunable Feshbach resonances [1, 2] have been performed, ranging from ultra-high resolution molecular spectroscopy [3] to the coherent coupling of atomic and molecular states [4] as well as the creation of bright matter wave solitons [5]. It also led to the production of new atomic [6] and molecular [7] Bose-Einstein condensates (BEC) and allowed to control pairing in ultra-cold fermionic gases [8].

Recently we demonstrated how atom-atom interactions in a ${ }^{87} \mathrm{Rb} \mathrm{BEC}$ can also be tuned with an optically induced Feshbach resonance [9] (see also [10]), a scheme which was originally proposed by Fedichev et al. [11, 12]. Optically induced Feshbach resonances offer advantages over magnetically tuned Feshbach resonances since intensity and detuning of optical fields can be rapidly changed. Furthermore complex spatial intensity distributions can be easily produced and optical transitions are always available even when no magnetic Feshbach resonances exist. A disadvantage of optically induced Feshbach resonance is the inherent loss of atoms due to excitation and spontaneous decay of the molecular state [9]. Typical lifetimes for excited molecular states are on the order of $10 \mathrm{~ns}$ which corresponds to a linewidth of $2 \pi \times 16 \mathrm{MHz}$. Evidently, coupling to molecular states with longer lifetime should improve the situation. Ground state molecules are stable against radiative decay, and narrow transition line widths on the order of $\mathrm{kHz}$ have been observed in two-photon Raman photoassociation [13, 14]. This raises the question whether it is possible to create optical Feshbach resonances using stimulated Raman transitions and whether this scheme might be advantageous compared to the one photon optical Feshbach resonance.

In this paper we indeed demonstrate that optical Feshbach resonances can be induced using a coherent two-color Raman transition to a highly vibrationally excited molecular ground state in $\mathrm{a}^{87} \mathrm{Rb} \mathrm{BEC}$. In the experiment we show how the scattering length and loss rates can be tuned as a function of the intensity of the lasers and their detuning from molecular lines. We use Bragg spectroscopy [15] as a fast method to measure the scattering length in our sample [9]. To fit and analyze our data we use a model by Bohn and Julienne [16]. We find that using the Raman scheme for optically induced Feshbach resonances leads to similar results in tuning of the scattering length as for the single photon Feshbach scheme. The Raman scheme does not lead to an improvement compared to the onephoton scheme because its atomic loss rate is not lower for a given change in scattering length. However, using a stimulated Raman transition does offer experimental advantages. To tune over the Feshbach resonance, the relative frequency of the two laser beams only has to be changed typically by several $\mathrm{MHz}$ which can be conveniently done using an acoustooptic modulator. This allows for very fast and precise control of the scattering length. On the other hand, working with a one-photon optical Feshbach resonance in the low loss regime typically requires large detunings and scan ranges on the order of GHz. The Raman scheme relaxes the necessity for absolute frequency control of the lasers which can be tedious to maintain far away from atomic lines. Since off-resonant light fields in general lead to dipole forces acting on the atoms, a variation of the scattering length via optical tuning leads to a variation of the dipole forces on the atomic sample. This unwanted effect can be made negligible for the Raman scheme which tunes over resonance within a small frequency range.

The paper is organized as follows: We start in section 11 by discussing the Raman scheme with a simple theoretical model. In section $\amalg$ we describe in detail our experimental setup and the measurement method. In section IV we discuss the experimental results which are compared with a theoretical model. The appendix gives details of the model that is used to describe the data.

\section{RAMAN SCHEME FOR OPTICAL FESHBACH TUNING}

Before discussing optical Feshbach tuning based on a twophoton Raman transition, it is instructive to briefly recall the one-photon scheme first [9, 11, 12]. This configuration uses 


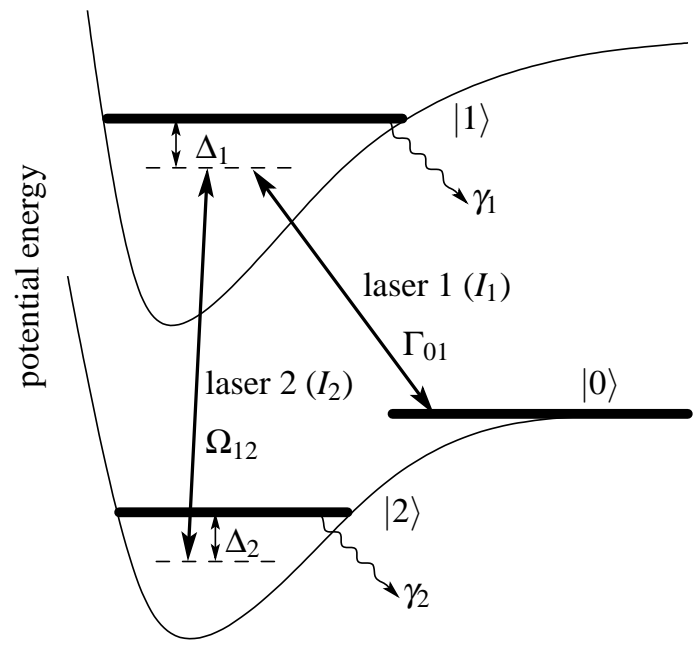

internuclear separation

FIG. 1: Schematic diagram of the transitions used for optically coupling the collisional state $|0\rangle$ to molecular states $|1\rangle$ and $|2\rangle .|1\rangle$ is electronically excited whereas $|2\rangle$ is in the electronic ground state. $\Delta_{1}$ and $\Delta_{2}$ are defined to be positive for the shown configuration.

a single laser beam tuned close to a transition from the scattering state of colliding atoms to a bound level in an excited molecular potential (states $|0\rangle$ and $|1\rangle$ in Fig. 11. Varying the detuning $\Delta_{1}$ or the intensity $I_{1}$ modifies the coupling and hence the scattering length. Atomic loss can occur through population of the electronically excited molecular state which has a decay width of $\gamma_{1}$.

Introducing a second laser as shown in Fig. 11will now couple the collisional state $|0\rangle$ to a bound level $|2\rangle$ in the ground state potential. As we will show, this allows for a tuning of the scattering length similar to the one-photon scheme. We now have, however, four parameters which can be used to influence the scattering length: the intensities $I_{1}$ and $I_{2}$ of lasers 1 and 2 and the detunings $\Delta_{1}$ and $\Delta_{2}$ as shown in Fig. 10 [23].

From [16, Eqs. (4.8)-(4.11)] one can extract approximate expressions for the inelastic collision rate coefficient $K_{\text {inel }}$ and the scattering length $a$ in a Bose-Einstein condensate [24]:

$$
\begin{gathered}
K_{\text {inel }}=\frac{2 \pi \hbar}{m} \frac{1}{k_{\mathrm{i}}} \frac{\Gamma_{01} \gamma_{1}}{\left(\Delta_{1}-\Omega_{12}^{2} / \Delta_{2}\right)^{2}+\left(\gamma_{1} / 2\right)^{2}} \\
a=a_{\mathrm{bg}}-\frac{1}{2 k_{\mathrm{i}}} \frac{\Gamma_{01}\left(\Delta_{1}-\Omega_{12}^{2} / \Delta_{2}\right)}{\left(\Delta_{1}-\Omega_{12}^{2} / \Delta_{2}\right)^{2}+\left(\gamma_{1} / 2\right)^{2}}
\end{gathered}
$$

Here $\Gamma_{01}$ denotes the on-resonance stimulated transition rate from $|0\rangle$ to $|1\rangle$ and is proportional to $I_{1} . \Omega_{12}$ is the Rabi frequency for the coupling of the states $|1\rangle$ and $|2\rangle$ and is proportional to $\sqrt{I_{2}} . \hbar k_{\mathrm{i}}$ is the relative momentum of the collision, where $\hbar$ is Planck's constant divided by $2 \pi . a_{\mathrm{bg}}$ is the background scattering length and $m$ is the atomic mass.

Equations (11) and (2) neglect spontaneous decay from state $|2\rangle\left(\gamma_{2}=0\right)$ and assume $\Gamma_{01} \ll \gamma_{1}$. Setting $\Omega_{12}=0$ yields the expressions for the one-photon Feshbach resonance as given in [9]. Equations (1) and (2) yield a Lorentzian and a corresponding dispersive line shape as a function of $\Delta_{1}$. In

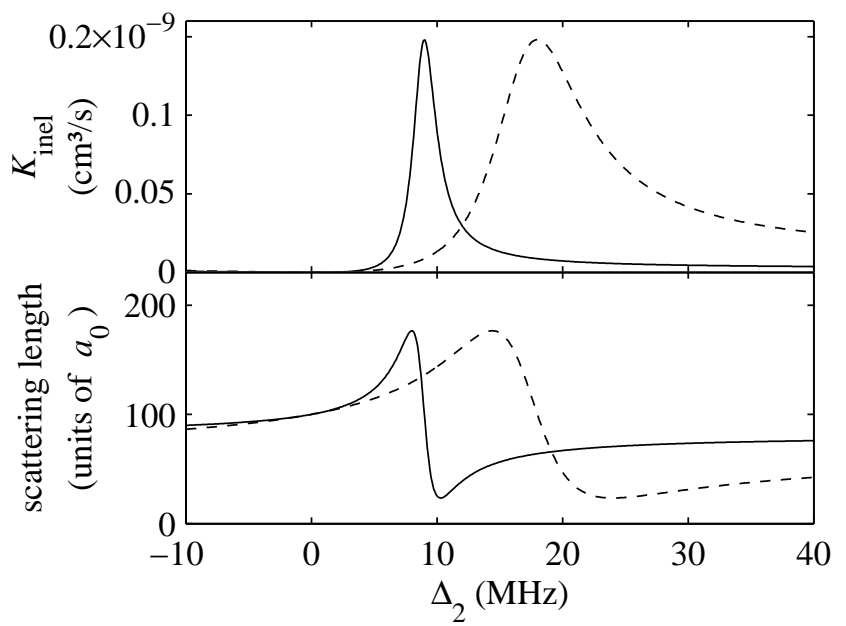

FIG. 2: $K_{\text {inel }}$ and scattering length $a$ according to Eqs. (1) and 2, plotted for two values of $\Delta_{1}$. Solid line: $\Delta_{1} / 2 \pi=100 \mathrm{MHz}$, dashed line: $\Delta_{1} / 2 \pi=50 \mathrm{MHz}$. The other parameters are $\Gamma_{01} / 2 \pi=50 \mathrm{kHz}$, $\Omega_{12} / 2 \pi=30 \mathrm{MHz}, \gamma_{1} / 2 \pi=25 \mathrm{MHz}$. The wavenumber $k_{\mathrm{i}}=2.5 \times$ $10^{5} \mathrm{~m}^{-1}$ corresponds to the finite size of the condensate wavefunction. $a_{0}$ is the Bohr radius.

our experiments, however, we hold $\Delta_{1}$ constant and scan $\Delta_{2}$. Figure 2 shows typical curves for $K_{\text {inel }}$ and $a$ for two detunings $\Delta_{1}$. The curves for $K_{\text {inel }}$ are slightly asymmetric, but for $\Delta_{1} \gg \Omega_{12}$ they can be well approximated by Lorentzians. This can be seen by expanding the denominator of Eq. (1) in terms of $\Delta_{2}$ at the resonance position. A light shift displaces the position of the resonance to $\Omega_{12}{ }^{2} / \Delta_{1}$. It is also interesting to note that the resonance width decreases with increasing detuning $\Delta_{1}$ as $\gamma_{1}\left(\Omega_{12} / \Delta_{1}\right)^{2}$.

In a sense the two-photon Raman-Feshbach resonance can be coined in terms of a one-photon Feshbach scheme. The detuning $\Delta_{2}$ effectively replaces the detuning $\Delta_{1}$ of the onephoton Feshbach scheme [25].

Since Eqs. (1) and (2) have exactly the same form as for the one-photon Feshbach resonance, it follows that, given a fixed free-bound transition rate $\Gamma_{01}$, the maximum tuning range of the scattering length for the two-photon case cannot be larger than in a one-photon scheme. Furthermore, given a fixed change-in-scattering-length, the loss rate as determined by $K_{\text {inel }}$ is not lower for the Raman scheme than for the onephoton scheme.

\section{EXPERIMENTAL SETUP AND METHODS}

\section{A. Production of BEC}

For the experiments we produce ${ }^{87} \mathrm{Rb}$ BECs of typically $1.2 \times 10^{6}$ atoms in the spin state $\left|F=1, m_{F}=-1\right\rangle$. Our setup comprises a magnetic transfer line [17] to transport atoms from a magneto-optic trap (MOT) chamber to a glass cell where the BEC is produced and all experiments are carried out. In a first step about $3 \times 10^{9}$ atoms are loaded within $4 \mathrm{~s}$ into a MOT directly from the background gas and are then 
cooled further to about $50 \mu \mathrm{K}$ in a molasses cooling phase. After optically pumping into the $\left|F=1, m_{F}=-1\right\rangle$ state we load the atom cloud into a magnetic quadrupole trap with a gradient of $130 \mathrm{G} / \mathrm{cm}$ in the (strong) vertical direction. Within $1.4 \mathrm{~s}$ the atoms are then moved via a magnetic transfer line [26] over a distance of $48 \mathrm{~cm}$ including a $120^{\circ}$ corner into a glass cell which is at a pressure below $10^{-11} \mathrm{mbar}$. In this cell we finally load the cloud into a QUIC trap [18] ending up with typically $4 \times 10^{8}$ atoms at a temperature of about $250 \mu \mathrm{K}$. All three coils of the QUIC trap are operated at a current of $40 \mathrm{~A}$, dissipating $350 \mathrm{~W}$. This results in trap frequencies of $\omega_{\text {radial }} / 2 \pi=150 \mathrm{~Hz}$ and $\omega_{\text {axial }} / 2 \pi=15 \mathrm{~Hz}$ at a magnetic bias field of $2 \mathrm{G}$. To achieve Bose-Einstein condensation we use forced radio-frequency evaporation for a period of $20 \mathrm{~s}$. The stop frequency is chosen so that we end up with condensates with a thermal background of about $25 \%$ of non-condensed atoms. At this value we concurrently get the highest number of atoms in the condensate and good reproducibility. For our measurements we consider only the condensed atoms.

\section{B. Raman lasers}

To realize the Raman scheme shown in Fig. 1 we use the electronically excited molecular state $|1\rangle=\left|0_{g}^{-}, v=1, J=2\right\rangle$ located $26.8 \mathrm{~cm}^{-1}$ below the $\left(S_{1 / 2}+P_{3 / 2}\right)$ dissociation asymptote [9, 19]. About $290 \mathrm{MHz}$ below the $J=2$ line, there is another rotational level with $J=0$ [27]. Although about five times weaker than the $J=2$ line, its effect cannot be totally neglected in our experiment. We choose level $|2\rangle$ to be the second to last bound state in the ground state potential. It has a binding energy of $636 \mathrm{MHz} \times h[13]$ where $h$ is Planck's constant.

The Raman laser beams are derived from a Ti:Sapphire laser using an acousto-optical modulator at a center frequency of about $318 \mathrm{MHz}$ in a double-pass configuration. This allows precise control of their relative frequency difference over several tens of MHz. Both Raman lasers propagate collinearly and are aligned along the weak axis of the magnetic trap (see Fig. 3. They have a $1 / \mathrm{e}^{2}$ waist of $76 \mu \mathrm{m}$, and their linear polarization is perpendicular to the magnetic bias field of the trap.

The Ti:Sapphire laser is intensity stabilized and its frequency has a line width of about $3 \mathrm{MHz}$. In order to stabilize its frequency relative to the photoassociation lines, the laser is offset locked relative to the $D_{2}$ line of atomic rubidium with the help of a scanning optical cavity. This yields an absolute frequency accuracy of better than $10 \mathrm{MHz}$. In all our experiments the Raman laser intensities were set to $I_{1}=300 \mathrm{~W} / \mathrm{cm}^{2}$ and $I_{2}=60 \mathrm{~W} / \mathrm{cm}^{2}$ at the location of the condensate, if not stated otherwise.

\section{Bragg spectroscopy}

To measure optically induced changes in the scattering length $a$, we use Bragg spectroscopy [9, 15]. This method allows for a fast measurement on time scales below $100 \mu \mathrm{s}$

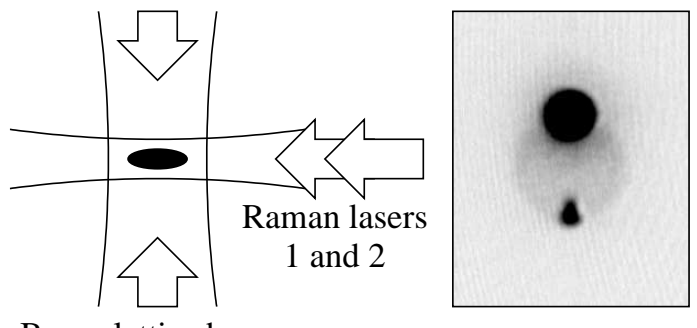

Bragg lattice beams

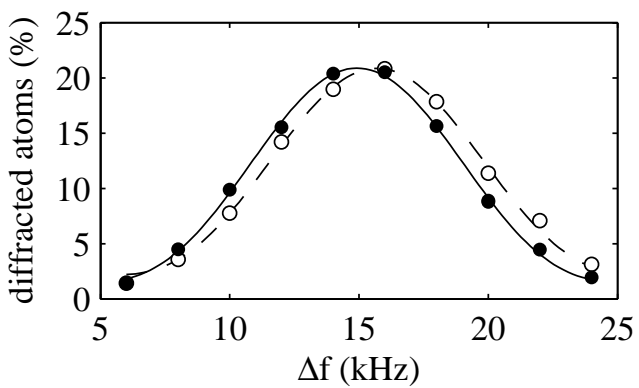

FIG. 3: Top left: Experimental arrangement of the laser beams (top view). Top right: Absorption image obtained after Bragg-diffracting a portion of the atoms to a state with a momentum of two photon recoils (lower atom cloud) and subsequent time of flight expansion. Bottom: Bragg resonance curves for two different relative detunings of the Raman lasers. The relative shift of $700 \mathrm{~Hz}$ is due to two different scattering lengths which are optically induced in the condensates. The atom numbers are the same for both curves. Shown is the percentage of diffracted atoms versus the frequency difference of the Bragg lattice beams. For better comparison we have scaled up the right curve by $10 \%$.

which is vital because of the rapid photoassociation losses we experience in our experiments. A moving lattice composed of two counter propagating beams with wavenumber $k$ and a frequency difference $\Delta f$ is used to diffract some of the condensate atoms to a state with non-zero momentum. When energy and momentum conservation are fulfilled, the Bragg lattice resonantly transfers a momentum of two photon recoils $2 \hbar k$ in a first order diffraction process. For the case of a homogenous condensate of density $n$, the resonance energy for Bragg diffraction is given by the sum of transferred kinetic energy $h \Delta f_{0}=(2 \hbar k)^{2} / 2 m$ and the change in mean-field energy $4 \pi \hbar^{2} n a / m$ [28]. This corresponds to a frequency difference of the Bragg lasers of

$$
\Delta f_{\mathrm{r}}=\Delta f_{0}+\frac{2 \hbar}{m} n a
$$

If the condensate is initially not at rest, the kinetic energy contribution $\Delta f_{0}$ to the Bragg resonance frequency (Eq. 3) contains an additional term $2 \hbar \mathrm{kp} / \mathrm{m}$, where $p$ is the initial atom momentum in the direction of the Bragg lattice. In our experiments we observe such a motional shift corresponding to condensate momentum of up to $p=0.1 \hbar k$. This momentum can partly be attributed to optical dipole forces of Raman beams which are slightly non-centered on the condensate. Partly it can be attributed to a forced oscillation of the condensate in the magnetic trap at $150 \mathrm{~Hz}$ which coincides 
with the trapping frequency. Since this oscillation is driven by a higher harmonic of the line frequency $(50 \mathrm{~Hz})$, it is in phase with the line frequency and we are able to stabilize the initial condensate momentum by synchronizing the experiment to the line. A stable initial condensate momentum can then be determined and canceled out by measuring $\Delta f_{\mathrm{r}}$ alternately for Bragg diffraction to the $+2 \hbar k$ and $-2 \hbar k$ momentum component. After these measures we were left with a residual momentum noise level of up to $p=0.01 \hbar k$.

In our setup the Bragg lattice beams are oriented along the horizontal direction perpendicular to the Raman laser beams (see Fig. 3) and have a width of $\approx 0.9 \mathrm{~mm}$. We extract both beams from a single grating stabilized diode laser and use two acousto-optical modulators to control the frequency difference. The laser is tuned $1.4 \mathrm{~nm}$ below the ${ }^{87} \mathrm{Rb} D_{2}$ line which defines $\Delta f_{0}$ in Eq. 3 to be $15.14 \mathrm{kHz}$. This frequency is much larger than the typical mean field contribution, $2 \hbar n a / m$, which in our experiments was below $3 \mathrm{kHz}$.

We illuminate the trapped condensate for $100 \mu \mathrm{s}$ with the Bragg lattice light. After $12 \mathrm{~ms}$ of free expansion the diffracted atoms are spatially separated from the remaining atoms. Absorption imaging allows us to determine the diffraction efficiency. By adjusting the Bragg laser intensity (typically $1 \mathrm{~mW}$ ) we keep the maximum diffraction efficiency between $15 \%$ and $20 \%$. When we scan the frequency difference $\Delta f$ and measure the fraction of Bragg diffracted atoms we obtain curves as shown in Fig. 3 (bottom). These curves have a width of approx. $9 \mathrm{kHz}$ as determined by the $100 \mu$ s length of our Bragg pulses. The shape of the curves is given by the Fourier transform of our square light pulses which we use to fit the data to obtain the resonance position $\Delta f_{\mathrm{r}}[9]$. The shift between the two Bragg spectroscopy curves in Fig. [3 (bottom) is optically induced by shining in the Raman lasers at the same time as the Bragg lattice. For both curves the atom numbers are the same and $\Delta_{1}=60 \mathrm{MHz}$. Only the Raman detuning $\Delta_{2}$ differs by $26 \mathrm{MHz}$. According to Eq. (3) this observed shift in Bragg resonance frequency is then due to a change in scattering length, induced by tuning $\Delta_{2}$. This demonstrates that we can tune the scattering length $a$ with a Raman Feshbach resonance.

\section{Determination of Scattering Length}

We use Eq. (3) to determine the scattering length $a$ from the measurements of the Bragg resonance frequency $\Delta f_{\mathrm{r}}$. Equation (3), however, is derived for the case of a homogenous condensate. Our trapped condensate, in contrast, which is subject to photoassociation losses exhibits a time and position dependent density $n$. This can be taken into account by replacing the density $n$ in Eq. (3) by an appropriate effective value $\bar{n}$.

A simple approach to estimate $\bar{n}$ is to calculate the spatial and time average of the condensate density $n$ over the duration of the Raman pulse length $T$. For this we use the rate equation for the local density $\dot{n}=-2 K_{\text {inel }} n^{2}$ for two-atom losses. The inelastic collision rate coefficient $K_{\text {inel }}$ governing this process is obtained from measuring the atom number at the beginning and the end of the light pulse. This procedure already yields good results which differ less than $10 \%$ from an improved approach which we use for our data analysis and which is explained in the following.

The improved approach consists of a full numerical simulation which describes Bragg diffraction in a dynamically and spatially resolved way. We divide the condensate into density classes and and treat their time dependence individually. The Bragg diffraction process is identified as a Rabi oscillation between a coherent two level system, i.e., the BEC component at rest and the Bragg diffracted component. The changing density of the condensate due to loss is reflected in a time dependent resonance frequency (see Eq. (3). As a result of these calculations we obtain for each density class a Bragg resonance curve similar to the experimental ones shown in Fig. 3 Averaging over these resonance curves and determining the center position yields the simulated value for the Bragg resonance $\Delta f_{\mathrm{r}}$. Using $\Delta f_{\mathrm{r}}=\Delta f_{0}+2 \hbar \bar{n} a / m$ we can then determine the effective density $\bar{n}$.

\section{RESULTS}

\section{A. Raman Scans}

Figure 4 presents measurements where the detuning $\Delta_{1}$ of laser 1 from the excited molecular state is set to $\Delta_{1} / 2 \pi=$ $60 \mathrm{MHz}$. The intensities of the Raman lasers 1 and 2 are $300 \mathrm{~W} / \mathrm{cm}^{2}$ and $60 \mathrm{~W} / \mathrm{cm}^{2}$, respectively. Fig. 4 a) shows the atom number after illuminating a condensate of initially $1.4 \times 10^{6}$ atoms for $100 \mu$ s with the Raman lasers. Scanning the Raman detuning $\Delta_{2}$ we find a strong loss of atoms on resonance. As already expected from Eq. (1) the line shape is slightly asymmetric. Figure 4 (b) shows the resonance frequency $\Delta f_{\mathrm{r}}$ as measured by Bragg spectroscopy. When we analyze the data in Fig. 4 a) and (b) with the improved procedure described in section 1 ID we obtain values for the scattering length which are shown in Fig. 4 (c). The scattering length $a$ shows a dispersive variation between $50 a_{0}$ and $140 a_{0}$ as we scan over the resonance. The dispersive scattering length curve is offset by about $20 a_{0}$ from the background scattering length $a_{\mathrm{hg}}=100 a_{0}$ for ${ }^{87} \mathrm{Rb}$ in the $\left|F=1, m_{F}=-1\right\rangle$ state $[20,21,22]$. This is due to the one-photon Feshbach tuning of laser 1 , in agreement with our previous measurements [9].

We find that Eqs. (1) and (2) are not sufficient to describe these data properly, mainly because they neglect the decay rate $\gamma_{2}$. A more complete model (see Appendix), also taking into account both the $J=0$ and $J=2$ rotational levels, was used for creating fit curves [29], depicted as solid lines in Fig. 4 The fact that the data for atomic loss as well as for the scattering length $a$ are both well described by the theoretical curves is an intrinsic consistency check for our model and our data analysis.

The shape of the signal $\Delta f_{\mathrm{r}}$ in Fig. 4 $\mathrm{b}$ ) is a combination of the effects of the varying scattering length $a$ and the varying atom number (see Eq. (3)). This is illustrated by the dashed and continuous lines in Fig. 4 (b): The dashed line shows the expected signal if only the variations in atom number would occur and the scattering length stayed constant [30]. The con- 


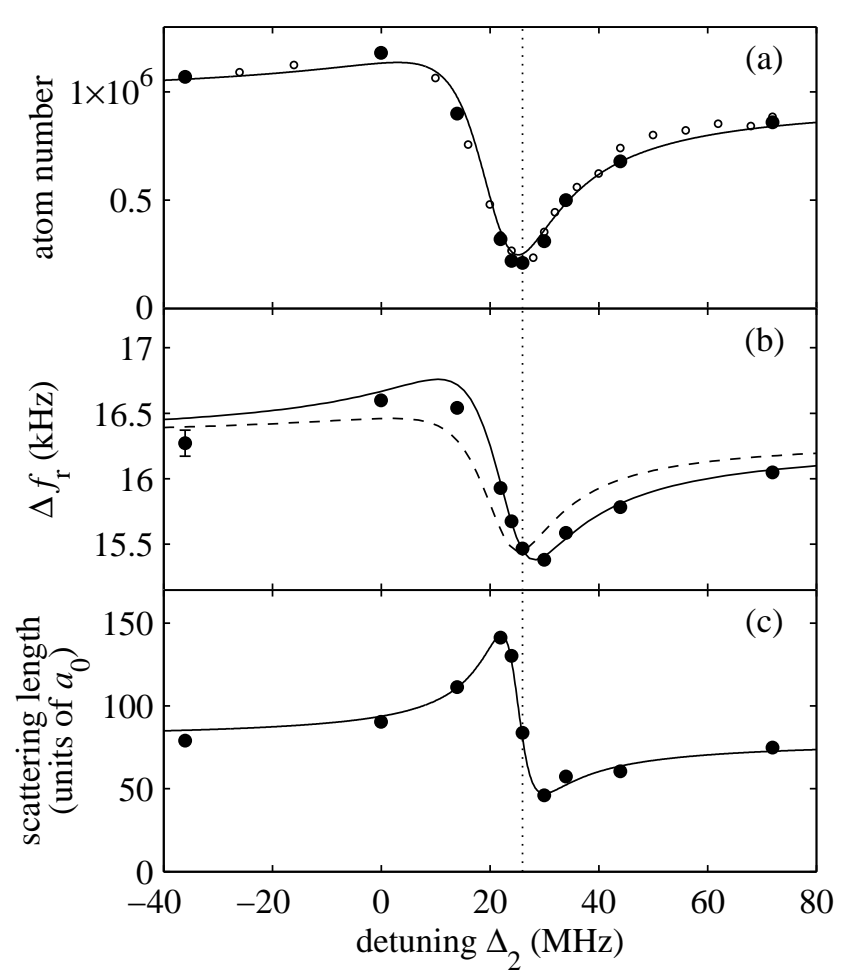

FIG. 4: Optical Feshbach resonance using a Raman scheme. (a) shows the measured atom number after the Raman pulse, (b) the measured Bragg resonance frequency and (c) the scattering length, as determined from (a) and (b). In (a) the filled circles correspond to measurements where Bragg spectroscopy was used to determine the scattering length, while the small open circles stem from additional loss measurements without Bragg spectroscopy. From our measurements we estimate the uncertainty of the Bragg resonance frequency to be smaller than $\pm 100 \mathrm{~Hz}$, as indicated by the error bar in (b). The solid lines in (a), (b) and (c) are from a model calculation (see Appendix and text). The dashed line in (b) shows the expected signal if there was only loss in atom number but no change in scattering length (see also discussion in text). The vertical line indicates the location of maximal loss in (a) and helps to compare the relative positions of the three curves.

tinuous line takes both the variations in atom number and in scattering length into account. The deviation of the measured data points from the dashed line is due to an optical induced change of the scattering length.

\section{B. Dependence on detuning}

We now investigate how detuning $\Delta_{1}$ affects the scattering length $a$. Figure 5 shows a set of three curves showing the scattering length for detunings $\Delta_{1} / 2 \pi=40,60$ and $90 \mathrm{MHz}$.

The measurements clearly show that the position and width of the resonances depend on $\Delta_{1}$. The change in position can be mainly explained as light shifts of levels $|1\rangle$ and $|2\rangle$ due to laser 2 . The decrease of the resonance width with increasing detuning $\Delta_{1}$ follows directly our discussion in section [ The solid lines are model calculations as described in detail

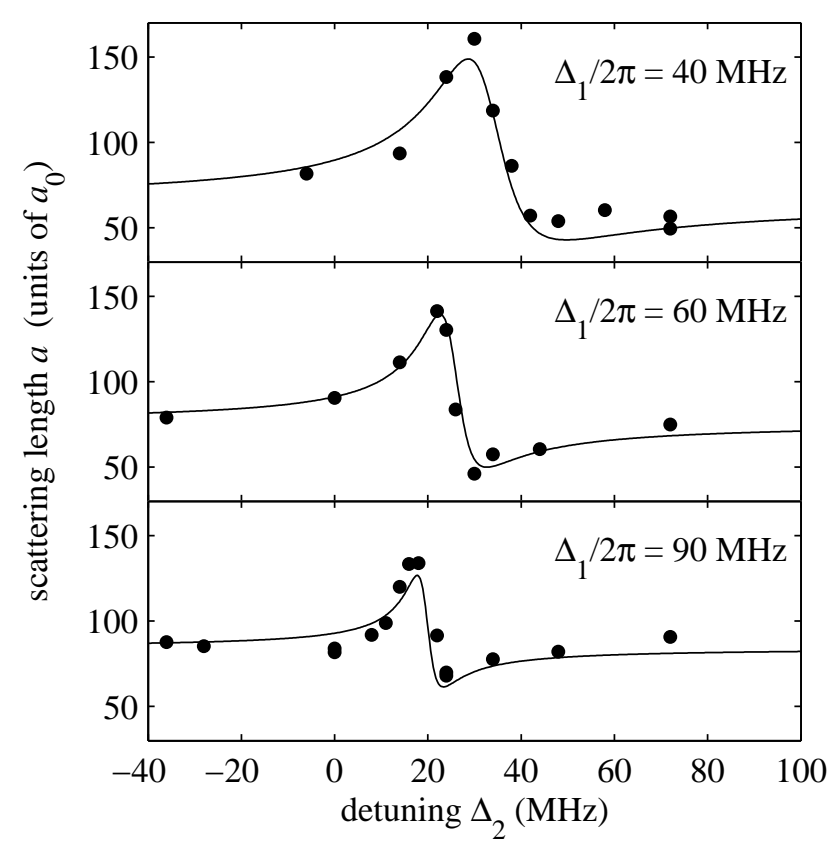

FIG. 5: Variation of the scattering length with Raman detuning for three various detunings $\Delta_{1}$ from the excited molecular state. The solid line is a calculation (see Appendix) which uses a single set of parameters for all curves.

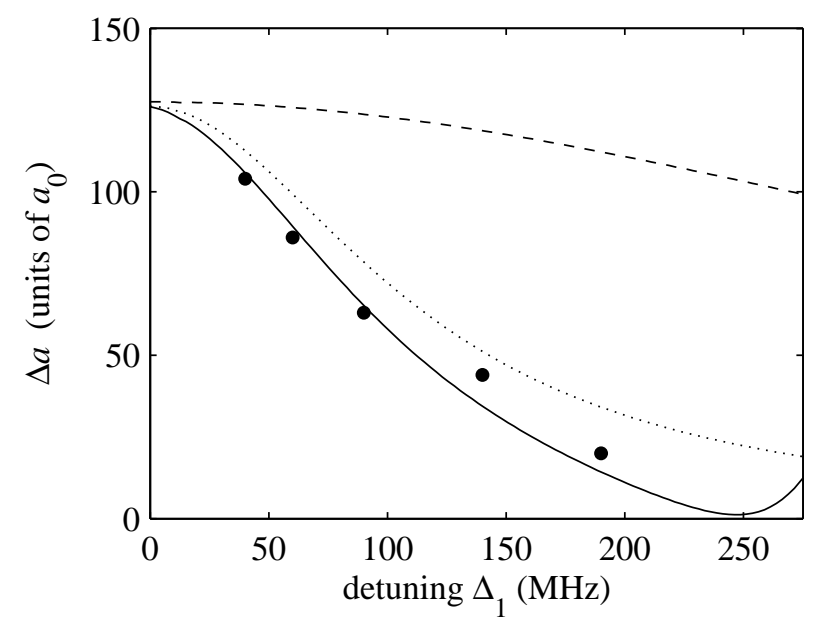

FIG. 6: Maximum variation in scattering length $\Delta a=a_{\max }-a_{\min }$ versus one-photon detuning $\Delta_{1}$. Solid line: full model calculation (see Appendix). Dotted line: 3-level model (see Fig. 1, with $\gamma_{2} / 2 \pi=2 \mathrm{MHz}$. Dashed line: 3-level model, with $\gamma_{2} / 2 \pi=100 \mathrm{kHz}$.

in the Appendix. They are derived from a simultaneous fit to the data shown in Fig. [5 and a large number of atom loss measurements with different detunings (not shown). The set of fit parameters is listed in Appendix A2 We also use this same set of parameters for the theoretical curves in Figs. 6 and 7

It is interesting to note from Fig. 5 that the amplitude of the dispersive scattering length signal decreases as $\Delta_{1}$ becomes larger. This is not to be expected from the simple model Eqs. (11) and (2). To investigate this effect we have performed 
scans for atom loss and scattering length for several detunings $\Delta_{1}$. Figure 6 shows the maximum variation in scattering length, $\Delta a=a_{\max }-a_{\min }$, obtained for detunings $\Delta_{1}$ ranging from $40 \mathrm{MHz}$ to $200 \mathrm{MHz}$. Here, $a_{\max }$ and $a_{\min }$ are the maximal and minimal scattering length values for corresponding scan curves. Typical scan curves are shown in Fig. 5 Each data point in Fig. 6 was derived from a complete scan and corresponds to one day of data collection.

An analysis of our data using our theoretical model indicates that the decrease of $\Delta a$ as a function of $\Delta_{1}$ is a consequence of two effects.

(i) To properly model these measurements we have to assign to the molecular state $|2\rangle$ in the ground state potential a non-negligible decay width $\gamma_{2} / 2 \pi \approx 2 \mathrm{MHz}$. For comparison, two calculations of a 3-level model are plotted in Fig. 6 For small $\gamma_{2} / 2 \pi=100 \mathrm{kHz}$ (dashed line) $\Delta a$ decreases only weakly. For $\gamma_{2} / 2 \pi=2 \mathrm{MHz}$ (dotted line) the theory fits the data much better. Such a large decay rate of a ground state level is surprising. It seems too large to be explained purely by collisions. We find that the decay rate increases with the light intensity. At low light powers of a few $\mathrm{W} / \mathrm{cm}^{2}$ we have observed very narrow linewidths $\gamma_{2} / 2 \pi$ on the order of a few $\mathrm{kHz}$, similar to the values reported by [13, 14]. The broadening of the molecular ground level could be due to coupling to excited molecular levels. We can exclude, however, from our experimental data that these levels are located within our experimental scanning range between the states $|1\rangle$ and $|3\rangle$. This would lead to additional resonance features in the scattering length, absorption and light shifts, which are inconsistent with our data. In contrast, our data indicate a relatively constant background loss rate of the ground level over the experimental scan range. This allows us to analyze the data successfully with our simple few-level model. Besides coupling to excited molecular states, we suspect that coupling to the $d$-wave shape resonance of the scattering channel also gives rise to a sizeable contribution to the molecular decay rate. Because the $d$-wave shape resonance is located very close (a few $\mathrm{MHz}$ ) to threshold, it is resonantly coupled to the molecular ground state level via the Raman transition. To include the shape resonance is beyond the reach of our simple model and has to be investigated later.

(ii) The second reason for the decrease in $\Delta a$ is a quantum interference effect involving both the $J=2$ and $J=0$ rotational levels as predicted by our model. At a detuning of $\Delta_{1} / 2 \pi \approx 250 \mathrm{MHz}$ the interference effect leads to a complete disappearence of the optical Feshbach resonance. We observe this in a corresponding disappearence of the atom loss feature in our measurements (not shown). The interference effect alone, i.e., without a $2 \mathrm{MHz}$ linewidth, is not sufficient to explain the experimental data in Fig. 6

\section{Dependence on intensity}

From the simple model Eq. 21 it is clear that the maximum variation in scattering length $\Delta a$ is proportional to $\Gamma_{01}$ and consequently scales linearly with the intensity $I_{1}$ of laser 1 . We have verified this dependence recently [9] for the case of

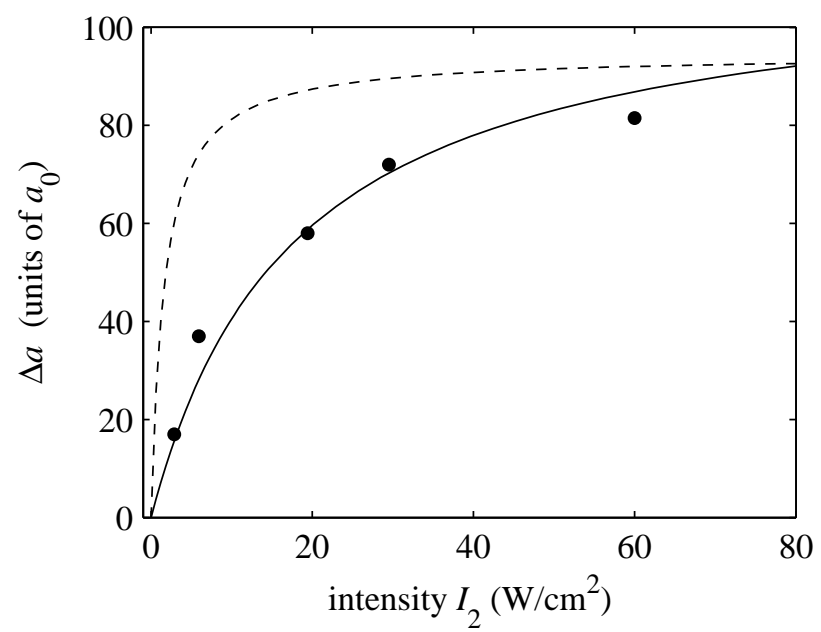

FIG. 7: Maximum variation in scattering length $\Delta a=a_{\max }-a_{\min }$ versus $I_{2}$. For this data set $I_{1}=300 \mathrm{~W} / \mathrm{cm}^{2}$ and $\Delta_{1}=60 \mathrm{MHz}$. The solid line is a full model calculation (see Appendix). The dashed line stems from the same model, but with $\gamma_{2} / 2 \pi$ set to $100 \mathrm{kHz}$ and is scaled by a factor of 0.84 for better comparison.

a one-photon optical Feshbach resonance.

In contrast, the dependence of $\Delta a$ on intensity $I_{2}$ of laser 2 is not so trivial. According to the simple model Eqs. (1) and (2) which neglect the decay rate $\gamma_{2}$, the maximum change $\Delta a$ is independent of $I_{2}$. It is also clear, that for $I_{2}=0$ we have $\Delta a=0$ since there is no dependence of scattering length on $\Delta_{2}$ at all. This unphysical discontinuous behavior can be resolved, if we introduce a finite decay rate $\gamma_{2}>0$. We then find that for increasing intensity $I_{2}, \Delta a$ rises continuously from zero to a value where it saturates. We observe this general behavior in our measurements presented in Fig. 7 Our full model, as described in the Appendix, describes the measured data well if we set the decay rate to $\gamma_{2} / 2 \pi=2 \mathrm{MHz}$ (solid line). In contrast, the dashed line in Fig. 7 shows the calculation for the same model where $\gamma_{2}$ is set to $\gamma_{2} / 2 \pi=100 \mathrm{kHz}$. Saturation then occurs at a much lower intensity $I_{2}$ than for $\gamma_{2} / 2 \pi=2 \mathrm{MHz}$.

\section{CONCLUSION}

Our experiments demonstrate the use of an optical Feshbach resonance for tuning of the scattering length via stimulated Raman coupling to a bound molecular state. Our results show that there is no advantage over a one-photon scheme when comparing tuning range and loss rate. However, for certain applications a Raman scheme is experimentally more favorable since it demands a lower tuning range of the lasers. Our presented theoretical model is in good agreement with our data and might be helpful when tailoring experimental parameters for a specific application. Furthermore it gives insight into the process of creating stable ultracold molecules via two-photon photoassociation. 


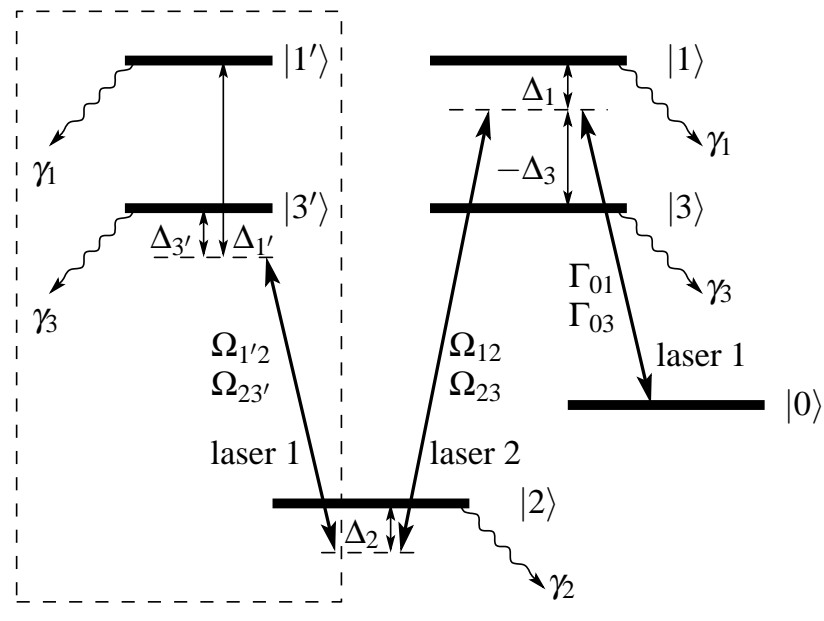

FIG. 8: Extended level scheme (compare to Fig1 for the 4-level model (right hand side) and its extension with 6 levels. State $|3\rangle$ corresponds to the $J=0$ level and lies $290 \mathrm{MHz}$ below the $J=2$ level $|1\rangle$. The 4-level model is based on levels $|0\rangle,|1\rangle,|2\rangle$ and $|3\rangle$. The auxiliary levels $\left|1^{\prime}\right\rangle$ and $\left|3^{\prime}\right\rangle$ are introduced in the extended model to describe the coupling between $|2\rangle$ and $|1\rangle,|3\rangle$, respectively, due to laser 1 (see text).

\section{ACKNOWLEDGMENTS}

We appreciate the help of George Ruff and Michael Hellwig at an early stage of the experiment. We thank Paul Julienne, Eite Tiesinga, John Bohn, Olivier Dulieu, Peter Fedichev, Andrea Micheli and Helmut Ritsch for valuable discussions. This work was supported by the Austrian Science Fund (FWF) within SFB 15 (project part 17) and the European Union in the frame of the Cold Molecules TMR Network under contract No. HPRN-CT-2002-00290.

\section{APPENDIX A: THEORETICAL MODEL AND FIT PARAMETERS}

We use a theoretical model by Bohn and Julienne [16] to fit the data in Figs. 4.7 In the following we give a short summary of this model and present the procedure to calculate the scattering matrix $S$, the loss coefficient $K_{\text {inel }}$ and the scattering length $a$. The model has the advantage that it is concise and intuitive and it allows treatment of multilevel systems with several couplings between the levels. The numerical calculations involve simple manipulations of small matrices.

In Fig. 8 the level scheme for our two models involving 4 and 6 levels are shown. We first restrict our description to the 4-level model as shown in the right part of Fig. 8 In this way our description stays compact and matrices are kept small. The extension to 6 or more levels follows the same scheme.

\section{The 4-level model}

Compared to Fig. 11 an additional excited level $|3\rangle$ is added. This level corresponds to the rotational level $J=0$ and lies $290 \mathrm{MHz}$ below the $J=2$ rotational level $|1\rangle[9]$. We work in the dressed atom picture and every level $|i\rangle$ is attributed a detuning $\Delta_{i}$ (see Fig. 8). $\Delta_{0}$ is arbitrarily set to 0 . The transition strengths from the continuum $|0\rangle$ to levels $|1\rangle$ and $|3\rangle$ are described by stimulated rates $\Gamma_{01}$ and $\Gamma_{03}$ which are proportional to the intensity $I_{1}$ of laser 1 . The transitions between the bound levels $|2\rangle$ and $|1\rangle,|3\rangle$ are characterized by the Rabi frequencies $\Omega_{12}, \Omega_{23}$, respectively, which are proportional to $\sqrt{I_{2}}$. Spontaneous decay from the bound levels leading to atomic losses is formally taken into account by introducing artificial levels $\left|\mathrm{a}_{i}\right\rangle$ for each level $|i\rangle$ to which a transition at rate $\gamma_{i}$ takes place (not shown in Fig. 8). All these couplings between different levels are summarized in the symmetric reaction matrix $K$. We arrange the level names in the order $\left(0, a_{1}, a_{2}, a_{3}, 1,2,3\right)$ and use them as row and column indices. The nonzero matrix elements of the $K$ matrix then read $K_{01}=\sqrt{\Gamma_{01} / 2}, K_{03}=\sqrt{\Gamma_{03} / 2}, K_{i a_{i}}=\sqrt{\gamma_{i} / 2}, K_{12}=\Omega_{12}$ and $K_{23}=\Omega_{23}$. Levels $0, \mathrm{a}_{1}, \mathrm{a}_{2}, \mathrm{a}_{3}$ are referred to as open channels, levels 1, 2 and 3 as closed channels. The reaction matrix $K$ is partitioned into open and closed channel blocks,

$$
K=\left(\begin{array}{cc}
\mathbf{0} & K^{\mathrm{oc}} \\
K^{\mathrm{co}} & K^{\mathrm{cc}}
\end{array}\right) .
$$

$K^{\mathrm{oc}}$ reads in our case

$$
K^{\mathrm{oc}}=\left(\begin{array}{ccc}
\sqrt{\Gamma_{01} / 2} & 0 & \sqrt{\Gamma_{03} / 2} \\
\sqrt{\gamma_{1} / 2} & 0 & 0 \\
0 & \sqrt{\gamma_{2} / 2} & 0 \\
0 & 0 & \sqrt{\gamma_{3} / 2}
\end{array}\right) .
$$

$K^{\mathrm{co}}$ is the transposed matrix of $K^{\mathrm{oc}}$, and

$$
K^{\mathrm{cc}}=\left(\begin{array}{ccc}
0 & \Omega_{12} & 0 \\
\Omega_{12} & 0 & \Omega_{23} \\
0 & \Omega_{23} & 0
\end{array}\right) .
$$

From $K$ the reduced $K$-matrix,

$$
K^{\text {red }}=K^{\mathrm{oc}}\left(D-K^{\mathrm{cc}}\right)^{-1} K^{\mathrm{co}}
$$

is calculated, eliminating the closed channels $1-3$, where $D$ denotes a diagonal matrix with diagonal elements $\left(\Delta_{1}, \Delta_{2}, \Delta_{3}\right)$. This determines the unitary $4 \times 4$ scattering matrix $S$

$$
S=\left(\mathbf{1}+i K^{\mathrm{red}}\right)\left(\mathbf{1}-i K^{\mathrm{red}}\right)^{-1} .
$$

From the matrix elements $S_{i j}$ of $S$ the trap loss coefficient $K_{\text {inel }}$ is calculated by

$$
K_{\text {inel }}=\frac{\pi \hbar}{\mu k_{\mathrm{i}}} \sum_{i}\left|S_{0 \mathrm{a}_{i}}\right|^{2}=\frac{\pi \hbar}{\mu k_{\mathrm{i}}}\left(1-\left|S_{00}\right|^{2}\right),
$$

where $\mu=m_{\mathrm{Rb}} / 2$ is the reduced $\mathrm{Rb}$ mass and $\hbar k_{\mathrm{i}}$ the relative momentum of the colliding atoms. The scattering length is obtained from $S_{00}$ via

$$
a=a_{\mathrm{bg}}-\frac{1}{2 k_{\mathrm{i}}} \frac{\mathfrak{I}\left(S_{00}\right)}{\mathfrak{R}\left(S_{00}\right)},
$$


where $\mathfrak{R}\left(S_{00}\right)$ and $\mathfrak{I}\left(S_{00}\right)$ denote the real and imaginary parts of $S_{00}$, respectively.

In the limit of small relative momentum $\hbar k_{\mathrm{i}}$ and small coupling strengths $\Gamma_{0 i} \ll \gamma_{i}, K_{\text {inel }}$ and the light induced change of scattering length $a-a_{\mathrm{bg}}$ are independent of $k_{\mathrm{i}}$ because the $\Gamma_{0 i}$ are proportional to $k_{\mathrm{i}}$ (Wigner threshold regime) [16].

\section{Extension of the 4-level model}

The 4-level model neglects that laser 1 (of which the intensity is typically five times greater than that of laser 2) also couples the levels $|2\rangle-|1\rangle$ and $|2\rangle-|3\rangle$. However, this coupling should be taken into account since laser 1 is not far detuned from these transitions (see Fig. 8 due to the small binding energy of state $|3\rangle(636 \mathrm{MHz} \times h)$ which is comparable to typical detunings $\Delta_{1}$. It mainly leads to broadening and light shifting of level $|2\rangle$. The additional coupling can approximately be taken care of by adding another two auxiliary levels $\left|1^{\prime}\right\rangle$ and $\left|3^{\prime}\right\rangle$ with detunings $\Delta_{1^{\prime}}=\Delta_{1}+\Delta_{2}+2 \pi \times 636 \mathrm{MHz}$ and $\Delta_{3^{\prime}}=\Delta_{3}+\Delta_{2}+2 \pi \times 636 \mathrm{MHz}$ as shown in Fig. 8 The coupling strengths $\Omega_{1^{\prime} 2}$ and $\Omega_{23^{\prime}}$ are fixed by $\Omega_{1^{\prime 2}}=\Omega_{12} \sqrt{I_{1} / I_{2}}$ and $\Omega_{23^{\prime}}=\Omega_{23} \sqrt{I_{1} / I_{2}}$. Compared to the 4-level model no new fit parameters are introduced. We can calculate $K_{\text {inel }}$ and the scattering length $a$ following the same recipe as for the 4-level model, only with larger matrices. Fitting the data in Figs. 4 [7 this extended model produced much better results than the 4-level model. For completeness we give here the fit parameters which were used in the calculations in Figs. 5$7\left(I_{1}=300 \mathrm{~W} / \mathrm{cm}^{2}\right.$ and $\left.I_{2}=60 \mathrm{~W} / \mathrm{cm}^{2}\right): \Gamma_{01} / 2 \pi=42 \mathrm{kHz}$, $\Gamma_{03} / 2 \pi=8 \mathrm{kHz}, \Omega_{12} / 2 \pi=32 \mathrm{MHz}, \Omega_{23} / 2 \pi=12 \mathrm{MHz}$, $\gamma_{1} / 2 \pi=25 \mathrm{MHz}, \gamma_{3} / 2 \pi=22 \mathrm{MHz}, \gamma_{2} / 2 \pi=2 \mathrm{MHz}$. We used $k_{\mathrm{i}}=2.5 \times 10^{-5} \mathrm{~m}^{-1}$. Due to the limitations of our model, these fit parameters should not be mistaken as the true values of the corresponding physical quantities.
[1] E. Tiesinga, B.J. Verhaar, and H.T.C. Stoof, Phys. Rev. A 47, 4114 (1993).

[2] S. Inouye et al., Nature 392, 151 (1998); Ph. Courteille, R.S. Freeland, D.J. Heinzen, F.A. van Abeelen and B.J. Verhaar, Phys. Rev. Lett. 81, 69 (1998); J.L. Roberts et al., Phys. Rev. Lett. 81, 5109 (1998).

[3] C. Chin, V. Vuletic, A.J. Kerman, and S. Chu, Phys. Rev. Lett. 85, 2717 (2000); A. Marte et al., Phys. Rev. Lett. 89, 283202 (2002).

[4] E.A. Donley, N.R. Claussen, S.T. Thompson and C.E. Wieman Nature 417, 529 (2002).

[5] L. Khaykovich et al., Science 296, 1290 (2002);K.E. Strecker, G.B. Partridge, A.G. Truscott, and R.G. Hulet, Nature 417, 150 (2002).

[6] S.L. Cornish, N.R. Claussen, J.L. Roberts, E.A. Cornell, C.E. Wieman, Phys. Rev. Lett. 85, 1795 (2000); T. Weber, J. Herbig, M. Mark, H.-C. Nägerl, and R. Grimm, Science 299, 232 (2003), published online 5 December 2002, 10.1126/science.1079699.

[7] S. Jochim et al., Science 302, 2101 (2003); M. Greiner, C.A. Regal, and D.S. Jin, Nature 426, 537 (2003); M. Zwierlein et al., Phys. Rev. Lett. 91, 250401 (2003); T. Bourdel et al., Phys. Rev. Lett. 93, 050401 (2004).

[8] C.A. Regal, M. Greiner, D.S. Jin, Phys. Rev. Lett. 92, 040403 (2004); M.W. Zwierlein et al., Phys. Rev. Lett. 92, 120403 (2004); C. Chin et al., Science 305, 1128 (2004), published online 22 July 2004, 10.1126/science.1100818.

[9] M. Theis et al., Phys. Rev. Lett. 93, 123001 (2004).

[10] F.K. Fatemi, K.M. Jones, and P.D. Lett, Phys. Rev. Lett. 85, 4462 (2000).

[11] P.O. Fedichev, Y. Kagan, G.V. Shlyapnikov, and J.T.M. Walraven, Phys. Rev. Lett 77, 2913 (1996).

[12] J.L. Bohn and P.S. Julienne, Phys. Rev. A 56, 1486 (1997).

[13] R. Wynar, R.S. Freeland, D.J. Han, C. Ryu, D.J. Heinzen, Science 287, 1016 (2000).

[14] T. Rom et al., Phys. Rev. Lett. 93, 073002 (2004).

[15] J. Stenger et al., Phys. Rev. Lett. 82, 4569 (1999).

[16] J.L. Bohn and P.S. Julienne, Phys. Rev. A 60, 414 (1999).

[17] M. Greiner, I. Bloch, T.W. Hänsch, and T. Esslinger, Phys. Rev. A 63, 031401(R) (2001).
[18] T. Esslinger, I. Bloch, and T.W. Hänsch, Phys. Rev. A 58, R2664 (1998).

[19] A. Fioretti et al., Eur. Phys. J. D 15, 189 (2001).

[20] Eberhard Tiemann, private communication.

[21] Paul Julienne and Eite Tiesinga, private communication.

[22] P.S. Julienne, F.H. Mies, E. Tiesinga, and C.J. Williams, Phys. Rev. Lett. 78, 1880 (1997).

[23] As we observe a significant light shift of level $|1\rangle$, depending on intensity $I_{1}$ of laser 1 [9], we measure the detuning $\Delta_{1}$ from the observed position of the one-photon line at a given intensity of laser 1 . Note that $\Delta_{1}$ is a one-photon detuning whereas $\Delta_{2}$ is a two-photon detuning.

[24] $K_{\text {inel }}$ is reduced by a factor of 2 as compared to the case of thermal atoms. This is because in a BEC all atoms share the same quantum state.

[25] There is even a more direct way to understand the two-photon Feshbach resonance in terms of a one-photon Feshbach resonance. Laser 1 couples the collision state $|0\rangle$ to a virtual level $\left|2^{\prime}\right\rangle$, which is generated by laser 2 acting on level $|2\rangle$. The splitting between $\left|2^{\prime}\right\rangle$ and $|1\rangle$ is given by $\Delta_{2^{\prime}}=\Delta_{1}-\Delta_{2}$. Its linewidth is $\gamma_{1}\left(\Omega_{12} / \Delta_{2^{\prime}}\right)^{2}$ and the transition rate $\Gamma_{02^{\prime}}=\Gamma_{01}\left(\Omega_{12} / \Delta_{2^{\prime}}\right)^{2}$.

[26] For our magnetic transport (similar to that described in [17]) 13 pairs of quadrupole coils are used. These transfer coils each have an inner diameter of $23.6 \mathrm{~mm}$, an outer diameter of $65 \mathrm{~mm}$, a height of $5.7 \mathrm{~mm}$ and consist of 34 windings. They are arranged in two layers above and below the vacuum chamber with a separation of $50 \mathrm{~mm}$. Peak currents of $75 \mathrm{~A}$ are necessary to maintain a vertical gradient of $130 \mathrm{G} / \mathrm{cm}$ during transfer.

[27] Due to different light shifts [9] for the $J=0$ and $J=2$ lines, their splitting is intensity dependent. The value of $290 \mathrm{MHz}$ is valid for an intensity of $300 \mathrm{~W} / \mathrm{cm}^{2}$.

[28] This is valid in the limit that only a small fraction of the condensate is diffracted.

[29] The resulting fit parameters are similar to those given in the Appendix.

[30] To account for the one-photon Feshbach tuning of laser 1, a value for the background scattering length $a_{\mathrm{bg}}=80 a_{0}$ was used for the calculation. 